\title{
Entrelacs
}

\section{Le jeu du Je masqué dans les films de Laurent Roth}

\section{Laurent Roth et Michèle Valentin}

\section{(2) OpenEdition}

\section{Journals}

Édition électronique

URL : http://journals.openedition.org/entrelacs/2938

DOI : 10.4000/entrelacs.2938

ISBN : 2261-5482

ISSN : 2261-5482

Éditeur

Éditions Téraèdre

Référence électronique

Laurent Roth et Michèle Valentin, «Le jeu du Je masqué dans les films de Laurent Roth », Entrelacs [En ligne], 15 | 2018, mis en ligne le 21 octobre 2018, consulté le 19 avril 2019. URL : http://

journals.openedition.org/entrelacs/2938; DOI : 10.4000/entrelacs.2938

Ce document a été généré automatiquement le 19 avril 2019.

Tous droits réservés 


\title{
Le jeu du Je masqué dans les films de Laurent Roth
}

\author{
Laurent Roth et Michèle Valentin
}

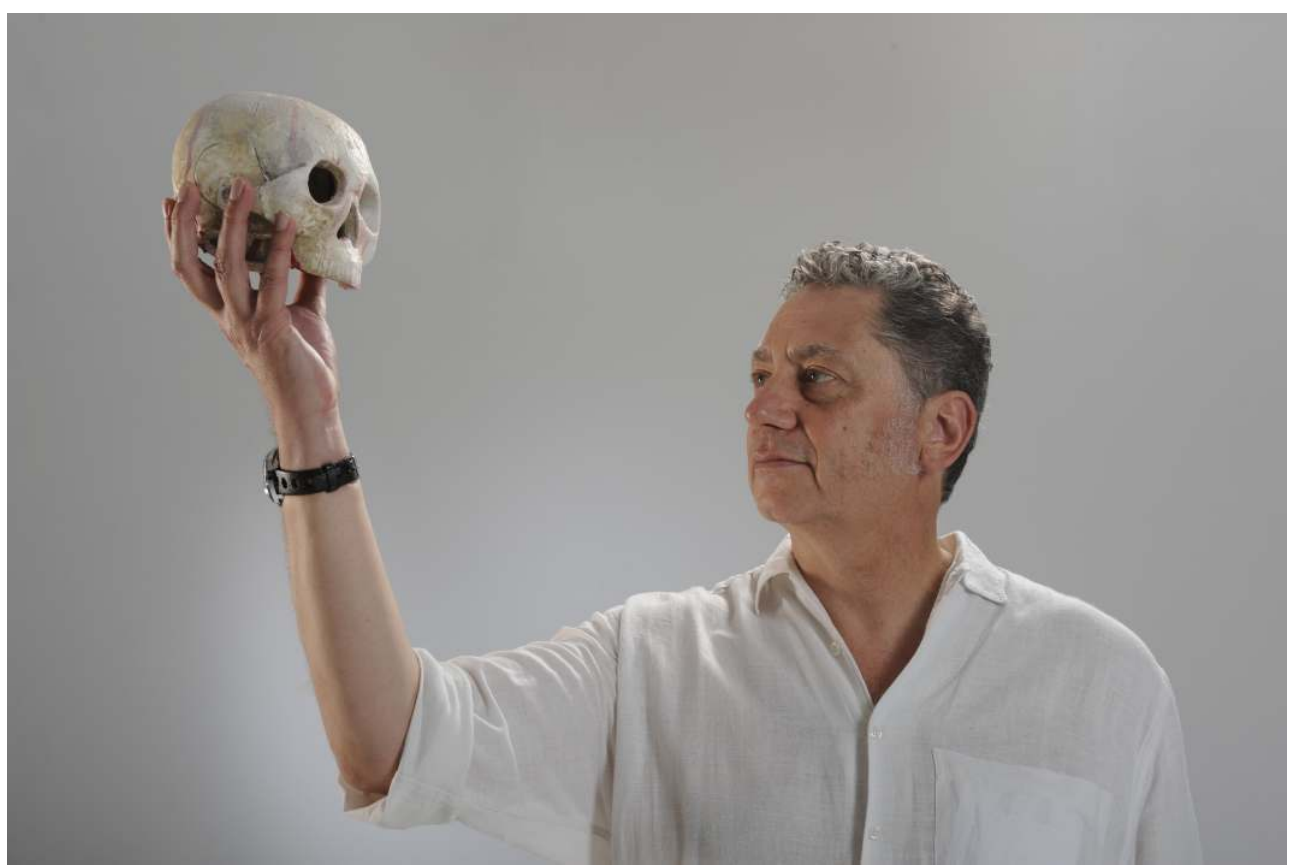

Laurent Roth en Hamlet @ C Henry Thurel

$1 \quad$ Le Jeu du Je masqué dans les films de Laurent Roth est un dialogue intime entre le cinéaste Laurent Roth et la psychanalyste Michèle Valentin pour parler du processus de création, et tenter de le démasquer. Tout au long de cet échange, les auteurs tentent de traduire la symbolique sans jamais l'enfermer. Elle est chaque fois une hypothèse, sinon une perspective de lecture sachant qu'il y en a forcément d'autres : un masque peut toujours en cacher un autre et c'est bien ce que nous allons découvrir. C'est là toute la valeur de création du cinéma en ce qu'il donne à voir autant qu'il dissimule. La tonalité du dialogue repose sur la déprise de tout savoir. Ni Laurent Roth ni Michèle Valentin ne savent à l'avance. Ensemble ils s'étonnent du jeu du Je-création. 

réalisateur, mais aussi celui de ceux qu'il met en scène. Si tu veux bien, nous nous arrêterons d'abord sur le décor de tes films de guerre où tu empruntes le masque de l'innocence. place : c'est elle qui incarne mon Je, ou plus justement cette part inconsciente du Je. Il était d'ailleurs prévu au départ que ce soit moi qui mène les entretiens avec les anciens reporters de guerre de l'armée française. Serge Daney ne s'y est pas trompé, puisqu'il parle de Mireille comme de mon « alter ego ", dans son très beau texte sur les Yeux brûlés ${ }^{1}$.

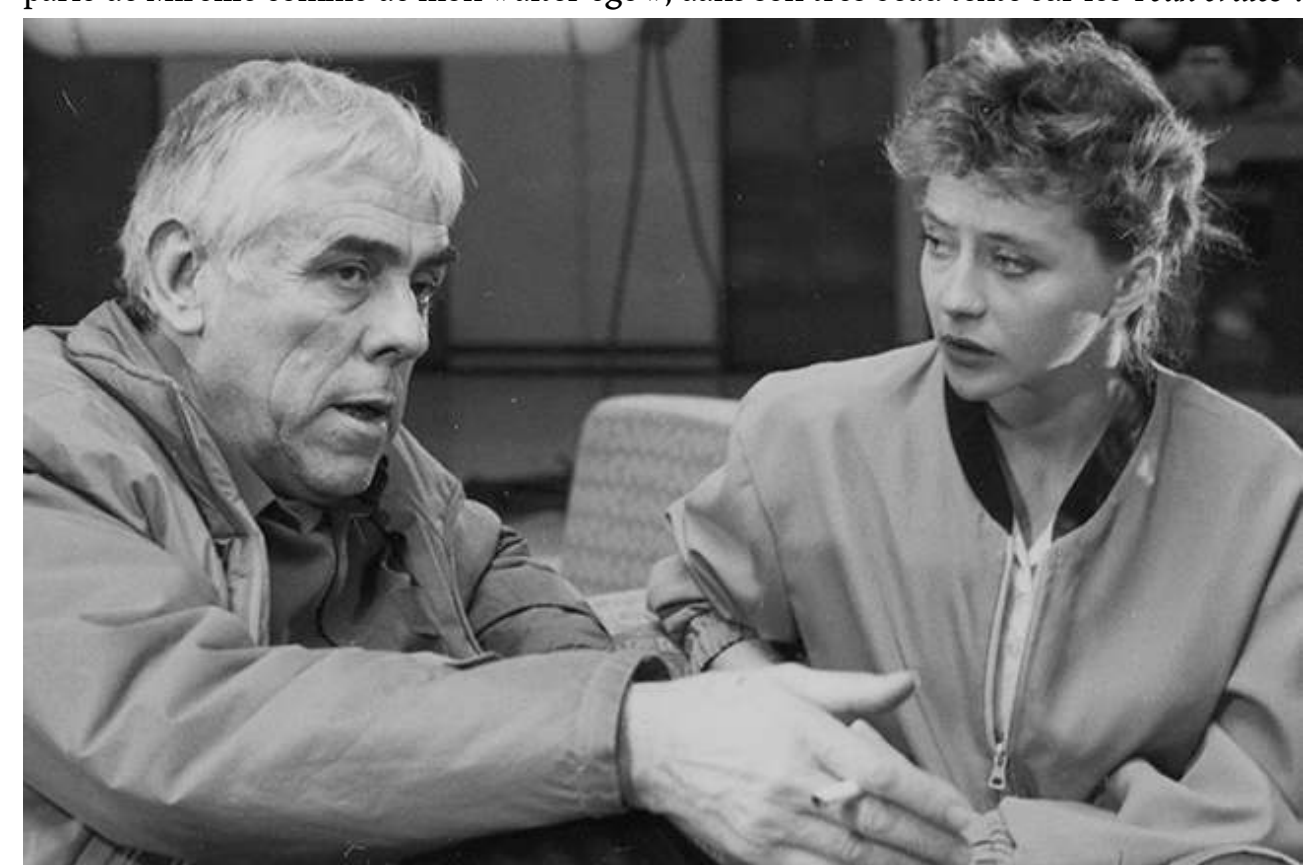

Mireille Perrier dans Les Yeux brûlés (1986)

6 Sans doute ton propos était de déléguer à Mireille ce Je, c'est d'ailleurs là le dispositif du film, mais il est possible que ce même Je, parce qu'il est du registre de la création, t'ait échappé de telle sorte qu'inconsciemment tu l'as projeté. Tu voulais cacher ton Je dans ton jeu et le Je de la création s'en est joué. Un tel processus oblige à se déprendre de la maîtrise. Pour un reporter de guerre cela ne peut qu'être désarmant. Tu les invites au risque et l'on voit dans le face à face avec Mireille combien les résistances sont perceptibles tant dans leurs propos que dans leurs attitudes. Ils luttent, progressent en tenue de camouflage pour, ultimement, échapper à l'embuscade. Toi, dans ce dispositif qui, en tant que dispositif ne t'échappe pas, pourquoi avoir éprouvé le besoin de te masquer?

7 Il m'était difficile d'apparaître dans ce film, commandé par une institution, l'Établissement cinématographique et photographique des armées, où je suis encore engagé quand j'obtiens le feu vert pour faire le film : en juillet 1985, je suis militaire. Je n'avais pas envie de me retrouver prisonnier de ma connivence avec eux. Passer par le féminin, celui d'une comédienne, c'était une manière d'être là incognito.

masque de la femme va donc avoir pour fonction de faire naître une parole déprise, faire entendre ce que ces reporters ont tant de mal à dire. Sous les traits de la femme se cache 
peut-être une sage-femme? Celle qui dévoile le discours, donne à entendre les non-dits, ces "dire en creux", révélant à l'insu du sujet-parlant ce qui ne se dit jamais à propos de la guerre. On voit des photos, des images, on lit ou entend des récits, mais rarement il n'est question de décrire le ressenti de ceux qui sont partie prenante de la guerre. Ultime pudeur?

Mireille Perrier est une femme qui dégage à la fois une puissance de vie et une forme d'incompréhension de la guerre. Mireille reste dans une vision d'enfant. Mes témoins sont obligés de s'expliquer comme des pères devant une enfant qui ne comprendrait pas. C'est en deçà de la sage-femme. Cela rejoint d'ailleurs Mireille dans son histoire personnelle, ses raisons intimes de faire le film, que je ne peux dévoiler ici.

Tu parles d'un Je en écho avec l'enfance. L'enfance, Marc Flament l'évoque, après avoir déclaré qu'il n'avait rien ressenti en photographiant un soldat mourant devant lui. C'est plus tard, dans l'évitement des associations, qu'il dit : "Je me suis mis à pleurer comme un gosse ". C'est quoi ce " comme un gosse" ?

11 À l'armée on dit « pleurer comme un gosse » ou « comme une fille ». Ce n'est pas viril de pleurer.

12 Le "comme un gosse " contiendrait toute la honte ressentie, alors même que c'est le Je qui pleure. Honte du Je ? Ou honte de n'avoir pu tout à fait renoncer au Je?

13 Oui, honte du Je dans un monde où c'est l'affirmation du Moi viril qui triomphe. Le Moi des Yeux brûlés, c'est plus qu'un Moi masqué : c'est un Moi casqué. Athéna porte un casque et elle sort de la cuisse de Jupiter. Athéna ne pleure pas. La question des larmes revient par deux fois dans les Yeux brûlés : lors de la confession de Marc Flament, dans la partie documentaire, et dans la fiction, lorsque Mireille s'enfuit de l'aéroport, à la fin du film. Ce dernier Je, le Je de la femme qui pleure, j'en suis responsable, je l'ai écrit dans le scénario de mon film, ce devait être la dernière image. On pourrait parler de "Je épiphanique ». Lorsque le Moi se brise, il pleure, il devient conscience du monde.

\section{Ce " Je qui pleure " de la comédienne, parle-t-il de tes larmes à toi ?}

15 Dans Les Yeux brûlés, c'est tout un jeu de cache-cache qui s'opère. Le cache-cache à propos de la guerre ne m'est pas tout à fait étranger. Mon père, rescapé de la déportation à Auschwitz, ne me parlait jamais de la guerre et lorsqu'au cinéma, en ouverture de séance, passaient les actualités, lors des scènes de guerre, il me mettait les mains sur les yeux dans le souci de me protéger. Ce n'est sans doute pas sans lien avec le titre de mon film: Les Yeux brûlés. Mes yeux à moi sont sans doute brûlés de n'avoir pas vu, et mes larmes sont encore loin de moi ; en 1986, je les confie à une comédienne.

Dans ce film on voit, on entend et on éprouve l'impact de la douleur et de ses enjeux dans un dispositif qui ressemble à une mise à l'épreuve. Comment ont réagi ces hommes de guerre lorsqu'ils ont vu le film terminé?

Ils ne l'ont pas aimé, voire détesté. Seul Marc Flament a fait exception, et c'est paradoxal puisque c'est celui qui semble le plus mis à l'épreuve par le dispositif des Yeux brûlés. Mais Flament est celui qui a accepté de souffrir, en faisant le récit de l'agonie de son camarade dans le djebel algérien, et des photos qu'il a prises à cette occasion. Le fait d'aller au bout de cet aveu pour lui a été cathartique ; sa femme m'avait confié à l'époque que cela avait été une libération. Et de fait, Marc Flament et moi sommes restés liés jusqu'à sa mort. Les autres, et en particulier Raoul Coutard et Pierre Schoendoerffer ont détesté être mis entre les mains de cette femme qui ne les a pas honorés comme ils le souhaitaient. Cette femme 
les a déshabillés. Elle parle d'une forme de jouissance. Elle les renvoie à l'intérêt subjectif qu'ils ont eu à faire leur métier, malgré l'uniforme. On ne parle jamais du plaisir personnel à faire la guerre, à participer de la guerre. Du plaisir solitaire qu'on tire de cette activité. La guerre fait jouir les hommes sinon ils ne la feraient pas. Et très souvent, elle les détruit.

Mireille les contraint à mettre en mot jouissance et souffrance. Elle les invite à une forme d'impudeur et c'est précisément ce que tu dévoiles. Ce nouage entre souffrance et jouissance est flagrant dans ton dernier opus: Pierre Schoendoerffer, La Peine des hommes (2017), qui est la reconstitution de l'intégrale de l'entretien de Pierre Schoendoerffer lors du tournage des Yeux brûlés.

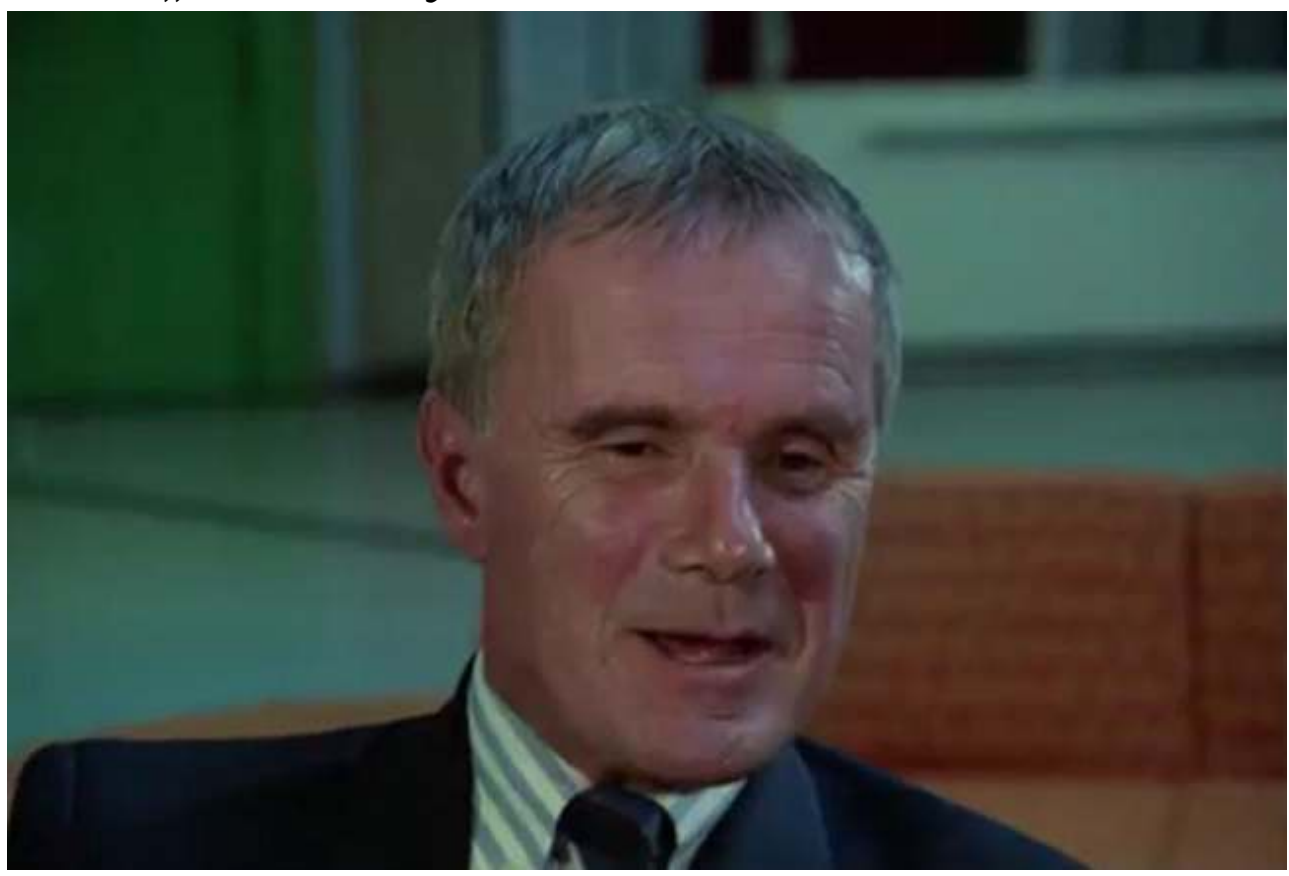

Pierre Schoendoerffer dans Pierre Schoendoerffer, La Peine des hommes (2017)

19 Oui, là, en une heure de film, on pourrait parler à la fois de la jouissance à revisiter une scène traumatique, et du conflit psychique que cela déclenche. C'est spectaculaire.

Le traumatisme se répète en boucle et cette boucle entrave le sujet, le fait disparaître. Le refoulé, dans Les Yeux brûlés, s'organise autour d'une malle, une malle qui revient plusieurs fois dans le film, attribut métonymique de l'homme disparu: Jean Péraud. Un Jean Péraud qui aurait sauvé la vie de Pierre Schoendoerffer, apprend-on dans Pierre Schoendoerffer, la Peine des hommes.

21 Péraud, c'est celui qui s'insurge parce qu'on n'a pas su protéger le jeune soldat qu'était Schoendoerffer, que l'on croyait mort. Ironie du sort, Schoendoerffer revient vivant d'Indochine, tandis que Péraud disparaît lors de leur évasion commune des camps vietminh. Son corps n'a jamais été retrouvé. Il incarne le perpétuel retour. Quelque part, dans l'au-delà de la malle, Péraud représente la perte inconsolable, le deuil impossible et la culpabilité de Schoendoerffer, celle qu'il tente en vain de masquer sous le regard inquisiteur de Mireille Perrier.

22 Cela explique sans doute la charge agressive de Schoendoerffer vis à vis de Mireille, qui l'oblige à se confronter à l'insupportable de la perte, l'insupportable de la dette. 
survie: dans ton film suivant L'Impromptu de Jacques Copeau (1993), il n'y a pas d'image filmée de Copeau, pas d'archive animée de lui. Ce n'est pas lui qui raconte sa survivance, mais ses disciples. Et dans la première image, c'est toi, le réalisateur qui apparaît de dos. Donc, on ne voit pas Copeau, mais toi de dos. Est-ce la première fois que tu apparais dans l'un de tes films?

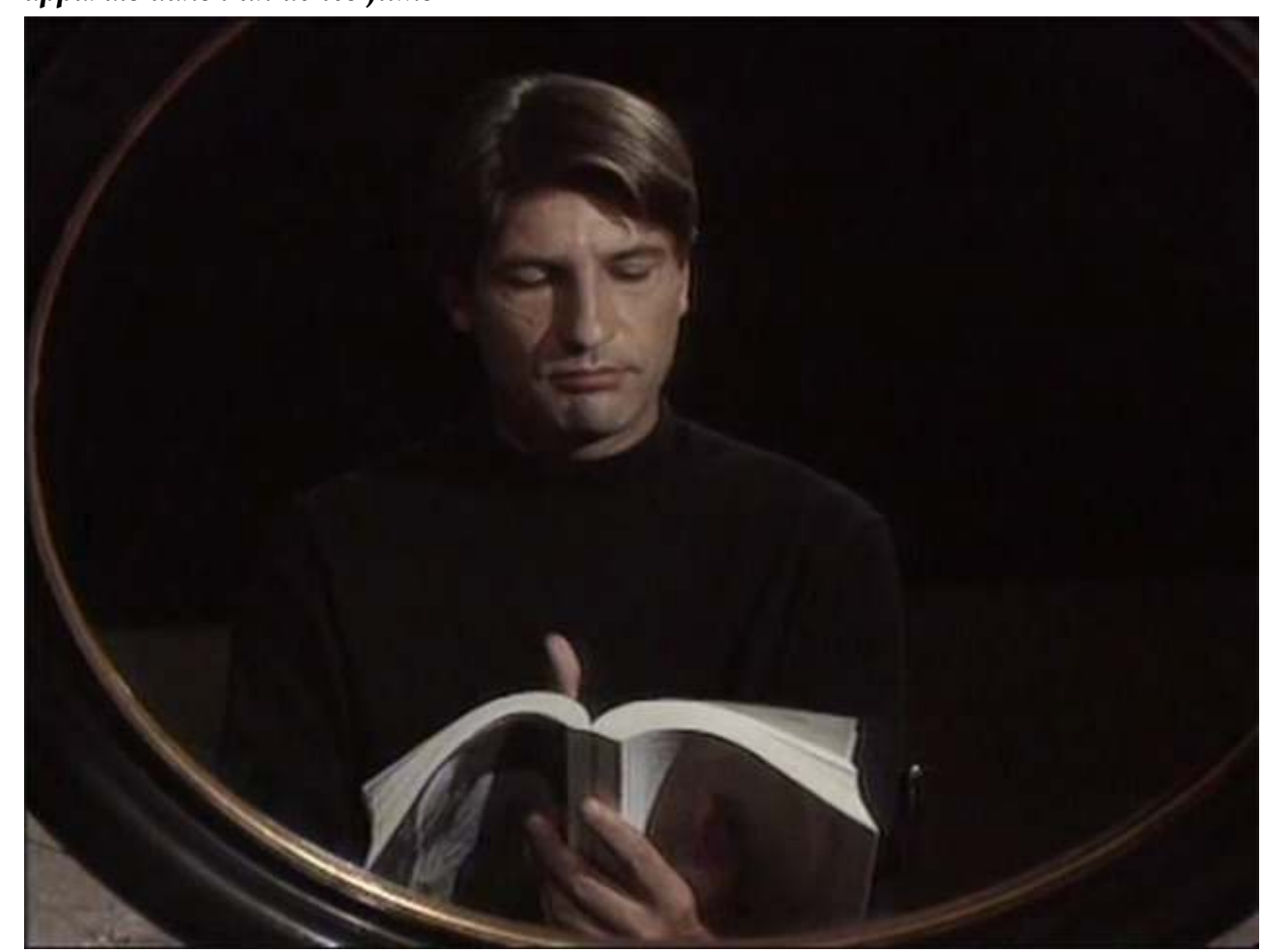

L'Impromptu de Jacques Copeau (1993)

Oui. Je suis le régisseur masqué, replié dans les gradins du décor que j'ai fait construire en studio. Je suis le meneur de jeu, qui est aussi un jeu de mémoire où chacun des comédiens introduit des fragments de la biographie de Copeau, leur "patron » comme ils disaient affectueusement: films d'archives, fragments de lettres, journaux intimes, extraits de pièces. Ils incarnent plusieurs facettes du personnage. Quant à moi, je ne prends jamais la parole et je n'apparais jamais de face.

On ne voit pas Copeau, mais on l'entend.

Oui, j'ai construit tout le film sur ses souvenirs qu'il a enregistrés à la fin de sa vie pour la radio. Cela lui donne une puissance spectrale très forte

\section{Une puissance comme dématérialisée?}

29 Sa voix sans corps va hanter le corps des autres. Copeau agit dans le corps des autres. Dans l'histoire du théâtre, tout le monde en parle comme d'un démiurge, d'une sorte de père fondateur. 


\section{n'est pas ici question d'incarner un homme, mais d'incarner ce qu'il laisse de vivant dans le} legs de sa recherche, de son travail. Une dimension transgénérationnelle?

Oui. Sur ce tournage, nous étions vraiment héritiers de Copeau au sens charnel : la petitefille de Jacques Copeau, Catherine Dasté, est un des quatre comédiens du groupe de travail que j'avais constitué pour le film, et beaucoup avaient travaillé avec elle, dans l'esprit du travail de Copeau. Moi même je les avais rejoints quelques années plus tôt comme auteur dramatique.

Et c'est curieusement dans la position de l'auteur que tu apparais à la toute fin du film.

33 Tandis que les comédiens jouent la scène finale du Petit Pauvre, l'ultime pièce de Copeau, je lis, visage masqué, je suis le lecteur des didascalies, j'apparais juste en soufflet dans l'image en haut à droite du cadre. Mon visage est masqué par le livre, seuls mes yeux regardent les acteurs sur le plateau. Je souhaitais prendre le masque pour rendre compte d'un cheminement intérieur. Celui d'un Copeau mystique et torturé.

Les didascalies sont une sorte de voix off, une voix à côté du texte. Donc, résolument, tu te situes dans cet à côté des comédiens, un à côté propre à Copeau. Charge aux comédiens d'incarner, dis-tu. Ce processus d'incarnation est fondamentalement différent de celui dont tu te sers dans Les Yeux brûlés où il s'agit de réveiller, révéler chaque personnage à luimême. Dans L'Impromptu de Jacques Copeau, l'incarnation va jusqu'à cette image extrême où Olivier Cruveiller qui joue Saint-François va allonger son corps sur l'ombre du Christ en croix projeté sur le tréteau nu du décor. Pourquoi la symbolique de la croix et que démasques-tu derrière cette symbolique?

Copeau s'était converti, et il va ressembler au Christ dans sa souffrance sur la Croix, dans bien de ses tentatives. Cette croix qui n'est qu'une ombre dans mon film représente sa volonté d'absolu, l'incarnation du Je supérieur par le théâtre.

Copeau prône un théâtre dépouillé. Quel lien entre le théâtre dépouillé et sa relation au Je dans la déprise du Moi?

Ce serait un théâtre sans auteur, issu d'un geste collectif, très actuel : une écriture de plateau, une écriture collective. Les Moi additionnés s'effacent dans un Je collectif. On sent très fortement cet héritage dans le jeune théâtre contemporain, où la notion d'auteur a quasiment disparu.

On t'a deviné sous les traits de Mireille Perrier dans Les Yeux brûlés et Pierre Schoendoerffer, la Peine des hommes, on te discerne en régisseur masqué pour l'Impromptu de Jacques Copeau et l'on te découvre enfin dans J'ai quitté l'Aquitaine (2005) détournant un test psychiatrique pour démasquer des enjeux de famille. La progression est nette: cette fois-ci tu apparais, tu incarnes, et tu es même de toutes les 
scènes! Et pourtant, tu n'es pas toi : tu prétends incarner un personnage souffrant, interné dans un asile. Ton masque a-t-il pour fonction de démasquer?

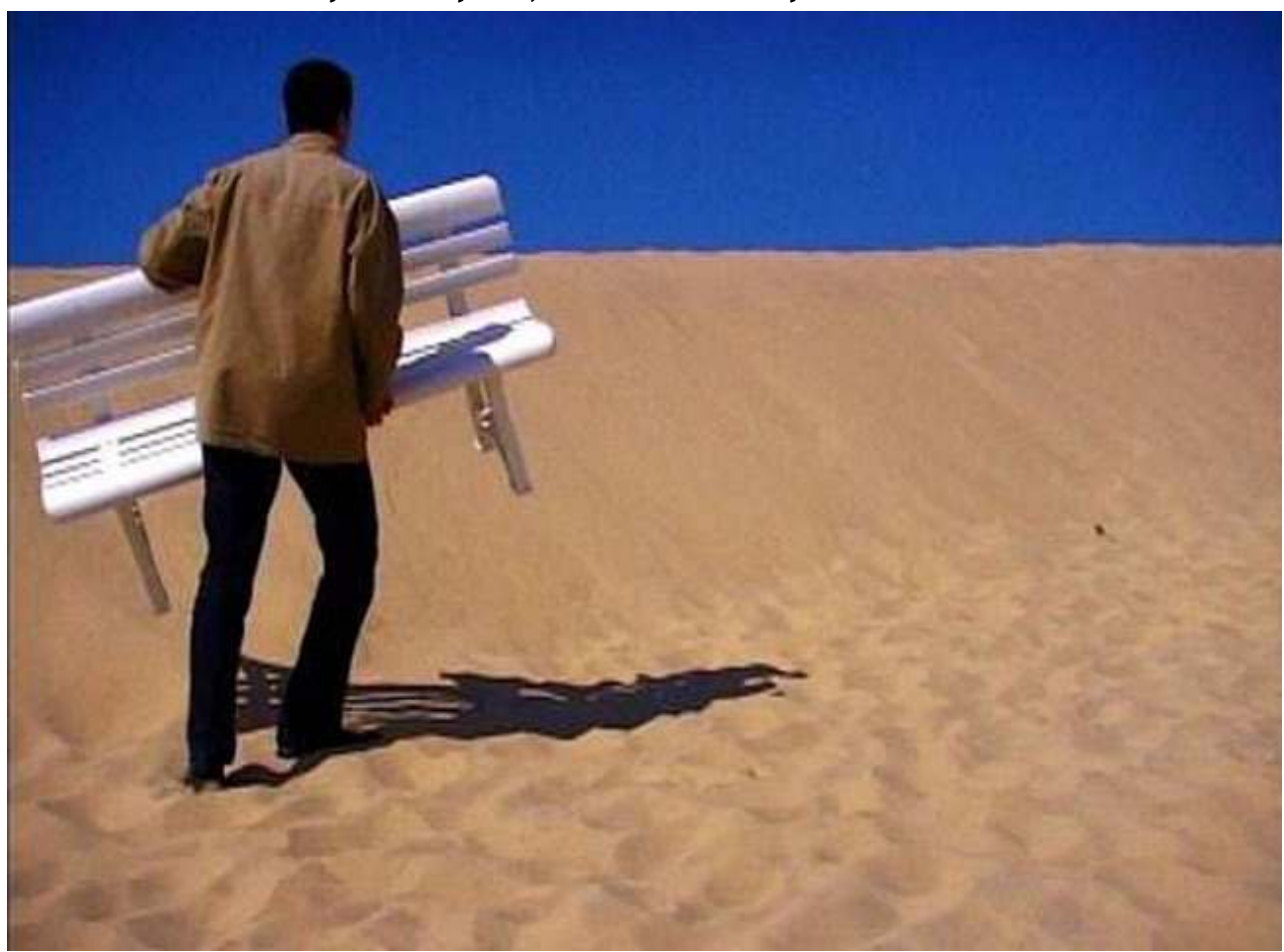

Laurent Roth dans J'ai quitté l'Aquitaine (2005)

39 Oui. J'ai pris conscience de la nécessité de jouer un personnage au cours même du tournage du film, où j'ai peu à peu stylisé ma présence à l'écran. Me masquer pour démasquer une construction du souvenir qui est officiellement celle du bonheur, la vulgate de toutes les familles : la maison, l'été, les vacances, les moments heureux... et derrière, des jalousies bien recuites, des chagrins indicibles. Comme dans Les Yeux brûlés, c'est un processus de mise à nu, mais cette fois-ci, c'est moi qui l'assume.

Dans ce film, J'ai quitté l'Aquitaine, tu invites les membres de ta famille à manipuler un test, qui, dans sa fonction, est détournée. On se demande alors forcément qui manipule qui et dans quel but?

41 C'est une stratégie révélatrice : il s'agit de se rendre compte que le territoire commun, celui de la maison, est perdu, le seul territoire commun est devenu le film, utopie de ce territoire disparu.

Tu révèles la douleur de la disparition, à la lumière de ta présence-absence. Tu regardes la souffrance, mais tu es autrement souffrant.

Dans la fiction du film, je suis le patient qu'on vient soigner. Alors qu'en tant que réalisateur, je suis le meneur du jeu. Il y a folie et maîtrise à la fois.

C'est ce que l'on retrouve dans tous tes films. Un personnage fou qui maîtriserait et révélerait la folie des autres. Dans J'ai quitté l'Aquitaine, tu tentes de déceler les dysfonctionnements à l'intérieur de la famille.

Oui, c'est impressionnant, le côté projectif du jeu a fonctionné à plein. Il y a des propos violents. Mon oncle disant que la véritable vocation de cette maison c'est d'être vide et d'y être seul. Comme le geste du cousin qui détruit la partie du domaine où nous 
habitions. Je pense que ma folie "prétendue » dans la fiction du film révèle celle des autres, mais elle les protège aussi, puisque ce sera toujours moi le plus fou dans l'affaire.

Toi, tu proposes une reconstruction de ce qui n'est plus, et forcément les propos des personnages de ta famille, dans l'après-coup, n'ont que vaguement à voir avec la réalité. Tu facilites donc un travail de reconstruction à partir du souvenir.

Pourtant tout le projet du test était de revenir sur l'ici et maintenant des lieux.

Mais les discours ne pouvaient être que de l'ordre de la reconstruction, donc jamais l'exactitude de la réalité.

Pour moi, le territoire de cet espace-temps du tournage et le test font que cet endroit a bien existé. Chacun en atteste et chacun le retrouve : il y a donc bien la tentation de retrouver une réalité. C'est cela, je crois, la puissance du cinéma.

C'est comme si, dans l'au-delà des discours projectifs, tu cherchais les non-dits accumulés au cours de ces temps partagés à l'époque.

Oui, en enfermant tout le monde dans ce dispositif, sans doute je cherche déjà ce dans quoi je suis enfermé, la question de l'origine, le secret caché.

Ce que tu ignores alors et que le Je de la création approche? Dans cette hypothèse, il y une certaine violence, un conflit psychique entre le Je-création approchant l'ignorance du Je du réalisateur que tu es. Peut-être est-il possible d'associer ton dispositif avec cette forme de violence. On repère l'agressivité dans la manière que tu as d'enfermer les membres de ta famille. Ils n'ont pas d'échappatoire. Depuis cette séquestration, tu leur suggères d'élaborer sur ce qu'ils ont pu ressentir d'un possible enfermement dans ce décor ritualisé de la maison de vacance familiale. Tu ne l'énonces pas sur le plan manifeste, mais le dispositif parle à ta place. C'est là que se situe la charge d'agressivité. On l'entend plus nettement dans la manière dont tu formules cette question à l'adresse de ta mère : "Si le domaine devait être attaqué, par qui serait-il attaqué?". C'est bien d'attaque dont il est ici question.

53 L'agressivité est là dès le premier plan: la famille réunie, toute la famille autour d'une table, avec ce lent panoramique qui les dévisage tous silencieux, et ma voix off qui raconte qu'ils ont décidé de m'enfermer. C'est en réalité un déplacement : c'est moi qui les enferme et leur impose silence à cet instant inaugural.

54 C'est très renversant, puisque tu apprendras plus tard que c'est finalement sur toi que pèse le silence dans le secret de famille. Finalement c'est d'un autre non-dit dont il est question dans J'ai quitté l'Aquitaine. Et les non-dits ont valeur de masques.

Oui, finalement la maison cachait la question des auteurs, de l'origine, de ma naissance.

Dans tes films, on pourrait dire que la question du Je-création démasquerait la dynamique de l'inconscient.

Oui, le jeu des masques m'amène à découvrir l'histoire de mon origine, mais à l'image du processus inconscient, il est protecteur, puisque la vérité apparaît travestie. S'inventer un personnage protège. L'ultime figure que j'invente dans J'ai quitté l'Aquitaine est un personnage libre, qui me permet de sortir de l'enfermement. C'est l'homme au banc; qui attire, tel un Orphée burlesque, les animaux à lui sur les rives de la Seine; et qui se transporte sur les dunes de sable, montant sans fin l'immensité qui croule sous lui. C'est une figure de l'errant, allégorie de la traversée du désert. À la fois le Christ et Sysiphe. Pour le coup je ne suis plus enfermé dans l'espace, mais dans une forme de la répétition du temps... 

radical que dans mes autres films puisqu'il est amplifié par la fixité photographique de chacun des plans. Au prologue, le personnage de l'Emmuré danse au milieu des autres, puis est brutalement isolé dans le confinement médical. Alors que dans J'ai quitté l'Aquitaine mon personnage est à l'écart, mais il mène le jeu.

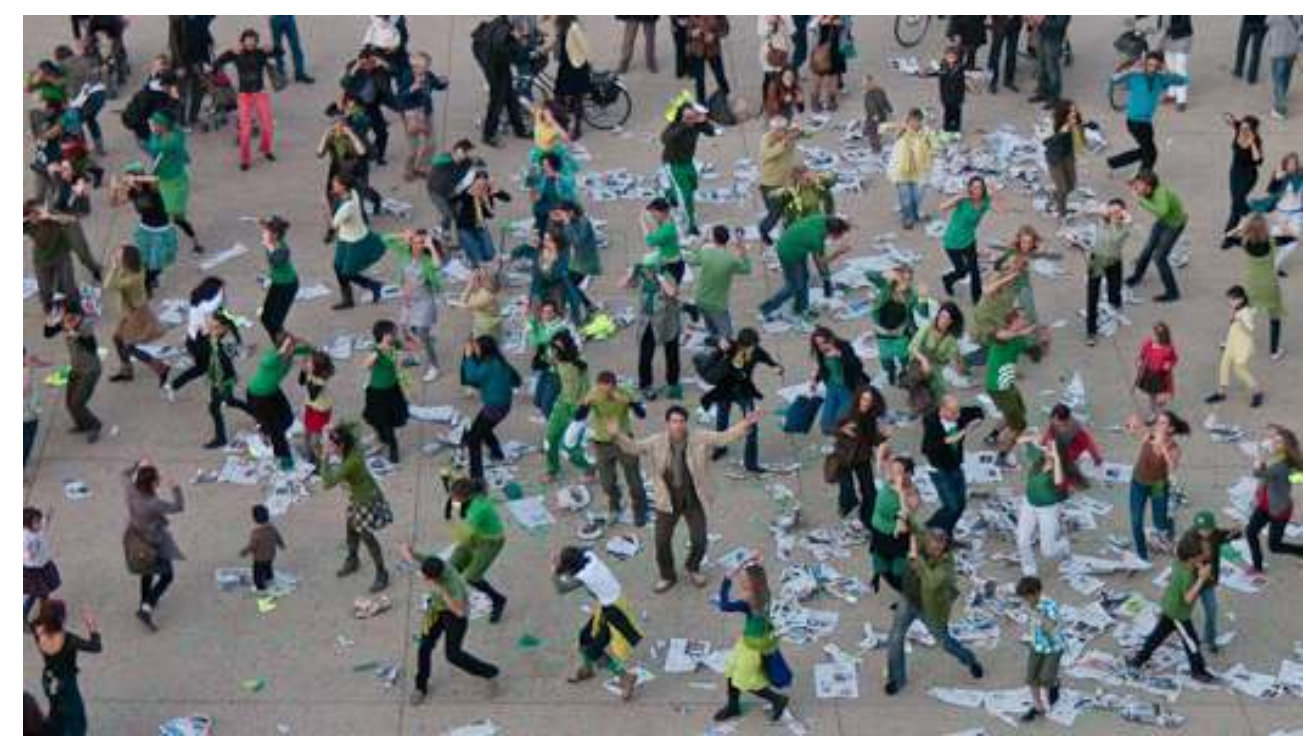

Le prochain film de Laurent Roth, L'Emmuré de Paris

Il y a dans L'Emmuré cette permanence du huis-clos que l'on retrouve dans tous tes films. Et en même temps, dans ce huis-clos formé par quatre rues de la Villette, il y a des masques, des figures, des "grands hommes", qui apparaissent et disparaissent. La tentative d'évasion de L'Emmuré est-elle une manière de quitter le masque?

C'est paradoxal. S'il y a évasion, elle passe effectivement par toute une série de «figures » qui sont autant de travestissements de mon personnage affligé de «manque de structure », de "manque de verticalité ». Le premier personnage est Blanqui, anarchiste et révolutionnaire: il est celui qui me permet de m'évader, lui que l'on appelait «l'Enfermé ».

C'est une évasion via l'identification puisque tu deviens "l'autre " au point de te quitter. Mais l'identification, est-elle un véritable moyen d'évasion dans le film ?

Oui, dans l'enveloppe charnelle d'un autre. Cependant, aucune enveloppe ne m'enferme. Ce sont des passages et c'est cette succession d'enveloppes qui me permet de naître en tout cas, à la fin. Passons en revue si tu le veux bien la série des « grands hommes » qui tous ont en commun d'être passé par ce quartier de la Villette qui est mon terrain d'observation dans L'Emmuré de Paris. Après Blanqui, c'est Pereire, l'instituteur des sourds muets, un philanthrope des Lumières qui crée le premier cimetière juif de Paris, dont je fais l'un des décors importants du film. 
Pereire, instituteur des sourds-muets, c'est aussi celui qui permet l'échange, qui permet qu'advienne une parole.

Oui. Il redonne la voix aux enfants muets, il fait rejaillir du son.

\section{C'est l'inverse de l'enfermement.}

Paradoxalement, pour m'évader, je m'enfuis dans l'espace clos du cimetière, une impasse, celle de l'enfermement. À la scène suivante: autre impasse, autre tentative, celle de l'évasion, avec la scène de la «nuit du phallus. » Une nuit où le Moi se retrouve face à luimême, dans une métamorphose animale, puis phallique...

\section{C'est une évasion onirique.}

Oui, mais dans cette nuit il n'y a plus personne. C'est l'évasion fantasmatique du sujet prisonnier du moi. Cette évasion survient trop tôt, le sujet n'est pas prêt. Elle est l'expression d'une puissance... D'une toute-puissance... Qui devient un jeu de masque grotesque.

Après, survient Louis XVI...

Là, dans l'épisode de la fuite à Varennes et du retour à Paris, je m'identifie à l'enfant de France, au Dauphin enfermé dans le carrosse... et dans le deuxième récit historique, c'est le maréchal Marmont qui apparait, lui même est enfermé dans Paris assiégé en 1814. Tous mes personnages sont finalement plus ou moins des personnages assiégés, enfermés. Sans parler de Ledoux qui va enfermer Paris.

2 Ledoux fait des portes, des guichets aux entrées de Paris.

Oui ! Un anonyme avait moqué son entreprise en placardant sur les murs de Paris: « Ce mur murant Paris a rendu Paris murmurant. » Aujourd'hui, Paris est muré par le mur de l'argent. Paris ne murmure même plus. Il digère...

\section{Quand quittes-tu le masque? Quand sors-tu psychiquement?}

J'incarne des figures marginales, jusqu'au bout je vais chercher les "grands hommes » pour échapper au regard surmoïque du Médecin-chef qui me juge, m'évalue et au fond me persécute. L'architecture des arcs, arceaux, arcades n'est que métaphore de la thérapie grandiloquente du Médecin-chef. Une thérapie où la grandeur de l'architecture a valeur d'écrasement du sujet, menace d'anéantissement. En sortir, trouver l'issue psychique, c'est en passer par le dépassement des grands hommes d'abord, du moi ensuite, pour accéder à l'issue finale du film, plutôt déconcertante...

76 Si je te comprends bien, la figure du Médecin-chef essaie d'affirmer une emprise dans l'ordre de l'espace, et ton personnage propose une issue dans l'ordre du temps et de la grande Histoire...

77 Oui, et c'est parce que ce Médecin-chef comprend que mon personnage tente une évasion dans la dimension historique qu'elle décide d'arrêter le temps, de l'anéantir... En arrêtant le temps, elle n'arrête que l'idée du temps linéaire : plus personne ne meurt.

Mais toi, est-ce qu'elle a le pouvoir de t'arrêter?

Moi, je suis dans le temps de la souffrance, celui du sujet, le temps qu'on ne peut pas normer. C'est le temps du Fou, du Christ, du Héros, de l'Idiot... Deux temps s'opposent, donc...

Ce hors temps que tu filmes à côté du temps arrêté, ce hors temps des saisons qui marchent à l'envers comme les gens, quel lien avec l'inconscient qui par essence est intemporel? 
81 À la fin du film, le temps est mélangé. Superposé. L'inconscient est à ciel ouvert... Et comme pour profiter du sommeil du Médecin-chef endormi dans le temps mathématique, mon personnage s'empare de Paris pour tout reconstruire, dans des travaux gigantesques, avec une puissance maléfique qui exclut et nivelle. Je m'empare de son Surmoi. projeté sur le Médecin chef auquel se substitue la figure de la vieille nonne, pour que le patient sorte de son statut de malade et laisse advenir ses émotions. De manière très paradoxale on assiste en image à un processus de régression qui, psychiquement, a valeur de maturation. En effet l'Emmuré se dés-emmure non pas en devenant un enfant, mais bien plutôt lorsqu'il parvient à avoir accès à l'enfant en lui. Cela a lieu dans la dimension transférentielle d'un autre, plus vieux, défait des enjeux sociétaux: la vieille nonne. L'intemporalité du film conduit à l'advenue du Je.

On assiste alors à une instrumentalisation de la masse.

architecturale, illustrée par les travaux de la Canopée des Halles, je deviens un grand homme en accomplissant le programme du Médecin-chef : transformer Paris en arcade commerciale.

Oui, mais tu instrumentalises autant que tu es instrumentalisé. De quel message s'agit-il ?

En « introjectant » le Moi du maître, on ne peut que devenir destructeur à son tour : à la fin du film, comme pour Job, toutes mes œuvres se déconstruisent sous mes yeux. C'est une gigantesque déconstruction du Moi, un effondrement du désir de toute puissance.

Qu'est ce qui dans le film accompagne le processus de déconstruction?

C'est la musique.

Dans les éléments qui parcourent le film, il y a aussi l'eau?

L'eau, c'est la fin de la musique. La musique et l'eau accompagnent la disparition du Médecin-chef qui s'évanouit, car ce n'est au fond qu'une chimère. Cette chimère s'éclipse pour laisser place à une fillette.

Quelle est la dernière image de toi ?

Une image, comme sortie d'un scenario d'heroic fantasy: une nonne se trouve là sur le pas de la porte du Je. Le patient entend, ça parle en lui...

Comment scandes-tu l'instant de la tombée des masques?

Dans la phrase finale : « Je suis cet enfant».

Si je récapitule ce mouvement de déprise: il faut la disparition du Moi, massivement Tel Zarathoustra s'adressant au soleil: «Il faut, comme toi, que je m'abîme, que je me couche pour employer le mot des hommes vers lesquels je veux descendre... Zarathoustra aspire à redevenir homme ${ }^{2}$ ».

$* * *$

Les masques tombent-ils vraiment au cinéma? Nous n'en sommes pas certains. Mais ce qui nous a semblé manifeste, c'est la dimension du "parlêtre » dans ce qu'il vient dire dans l'au-delà du dire et par là même, participe dans le dialogue, du dévoilement du Je dans l'intention d'un film.

Dans les propos des films que nous venons de citer, les masques ressemblent à des poupées russes. Après en avoir découvert une, on en découvre une autre, et puis une 
autre encore, la dernière est celle de l'enfant, l'enfant en soi et nous nous garderons bien d'aller plus loin, comme pour nous préserver du vacillement, cette béance de l'inconscient, dont Lacan disait: «Croyez bien, d'ailleurs, que moi-même je ne la rouvre jamais qu'avec précaution ${ }^{3} . . . »$.

\section{Résumés des films discutés}

- Les Yeux brûlés, 59', 1986. Production : Ecpad. Distribution, édition DVD : Shellac.

http://www.film-documentaire.fr/4DACTION/w_fiche_film/3463

Une jeune femme vient chercher à l'aéroport de Roissy une cantine militaire qui lui est retournée. Il s'agit des effets et clichés de Jean Péraud, reporter photographe disparu à Dien Bien Phu le 8 mai 1954. Bientôt, la discussion s'engage entre la jeune femme et les anciens compagnons de presse de Péraud qui sont présents. À travers les souvenirs et récits qu'elle suscite, ressurgissent les questions toujours actuelles sur ce qui fait l'image de guerre : le reporter est-il témoin ou combattant? Se protège-t-il des effets de la guerre en la filmant ou bien au contraire prend-il plus de risques pour rendre son témoignage? Peut-on parler d'art devant cette image faite face à la mort?

- L'Impromptu de Jacques Copeau, 58', 1993. Production: Agat Films. Distribution: Doc \& Films international.

http://www.film-documentaire.fr/4DACTION/w_fiche_film/437_0

Un studio plongé dans l'obscurité, un tréteau nu, quatre chaises, une table, un magnétophone, un projecteur, quelques livres et photos : voici les éléments de la machine à mémoire qui brossent un portrait vivant du «Stanislavski » français. En régisseur replié dans les gradins, Laurent Roth dirige quatre comédiens (dont Catherine Dasté et Marie Rivière) qui interprètent les textes que Jacques Copeau a laissés sur la scène. Des témoignages radiophoniques et filmés, pour certains inédits, viennent préciser les contours d'une figure qui, à travers l'histoire du théâtre du Vieux Colombier, continue de marquer en profondeur la vie théâtrale contemporaine.

- J'ai quitté l'Aquitaine, 53', 2005. Production: Cauri Films. Distribution DVD: La Huit (parution décembre 2018).

http://www.film-documentaire.fr/4DACTION/w_fiche_film/14229_0

Un réalisateur interné dans une institution psychiatrique tente de trouver la guérison en convoquant l'ensemble des membres de sa famille pour un test collectif : il s'agit, à l'aide d'une boîte de jeu de construction et de bobines de films de famille, d'essayer de restituer le plus exactement possible le bonheur tel qu'il était dans la maison de famille, disparue il y a maintenant vingt-cinq ans. Si un seul membre de la famille réussit à tout restituer, le patient pourra guérir... Sauf que rien ne se passe comme prévu.

Pierre Schoendoerffer, La Peine des hommes, 58', 2017. Production : Ecpad. Distribution, édition DVD : Shellac (bonus du dvd des Yeux brûlés)

http://www.film-documentaire.fr/4DACTION/w_fiche_film/51972_0

Intégrale de l'entretien de Pierre Schoendoerffer avec Mireille Perrier lors du tournage des Yeux brûlés, reconstitué d'après les rushes sonores du film et selon une méthode de restauration expérimentale et inédite : l'auteur de la 317 ème Section et du Crabe Tambour y commente avec passion le métier de la guerre, l'art de la filmer, et le sort de son frère d'arme Jean Péraud disparu à Dien Bien Phu. Durant cet entretien, en parlant à une 
femme, jeune et comédienne, on comprend très vite qu'il s'agit peut-être aussi d'une autre guerre pour Pierre Schoendoerffer : la guerre des mémoires, et la guerre des sexes... - L'Emmuré de Paris, 75', 2018. Production : Magnolias Films, La Huit. Distribution en cours. Post-scriptum à la Jetée de Chris Marker dans le Paris post attentats, L'Emmuré de Paris est la nouvelle fantaisie documentaire de Laurent Roth. L'acteur-réalisateur lâche aujourd'hui son personnage asilaire dans le quartier de La Villette pour une enquête dont le thème apparent touche à l'absurde : le recensement méthodique des arcs, arceaux et arcades dans l'architecture du quartier... Un prétexte pour parler de ce Paris où la mixité sociale est encore une réalité : un Paris au bord de sa disparition?

\section{NOTES}

1. Serge Daney, « Les Yeux brûlés, ou la métaphysique guerrière », Libération, 1986. Repris dans La Maison cinéma et le monde, tome 3 : les années Libé (1986-1991), P.O.L., 2012.

2. Friedrich Nietzsche, Le Gai savoir, «Incipit Tragoedia », fragment 342, 1882.

3. Jacques Lacan, Séminaire XI : Les quatre concepts fondamentaux en psychanalyse, 1964.

\section{RÉSUMÉS}

Le Jeu du Je masqué dans les films de Laurent Roth est un dialogue intime entre le cinéaste Laurent Roth et la psychanalyste Michèle Valentin pour parler du processus de création, et tenter de le démasquer. Tout au long de cet échange, les auteurs tentent de traduire la symbolique sans jamais l'enfermer. Elle est chaque fois une hypothèse, sinon une perspective de lecture sachant qu'il y en a forcément d'autres : un masque peut toujours en cacher un autre et c'est bien ce que nous allons découvrir. C'est là toute la valeur de création du cinéma en ce qu'il donne à voir autant qu'il dissimule. La tonalité du dialogue repose sur la déprise de tout savoir. Ni Laurent Roth ni Michèle Valentin ne savent à l'avance. Ensemble ils s'étonnent du jeu du Je-création.

Le jeu du Je masqué dans les films de Laurent Roth is an intimate dialogue between the film-maker Laurent Roth and the psycho-analyst Michèle Valentin in order to talk about the creative process and try to unmask it. All along that exchange, the authors try to translate the symbolic system without ever locking it up. Each time it is an hypothesis, if not a reading perspective knowing that there are necessarily others: a mask can hide another one and that's precisely what we are going to discover. That's where the creative value of the cinema lies in so far as it exposes as much as it conceals. The tonality of the dialogue rests on the giving up of any set knowledge. Neither Laurent Roth nor Michèle Valentin know anything in advance. Together they marvel at the game of the I-creative. 


\section{AUTEURS}

\section{LAURENT ROTH}

Scénariste, il a collaboré aux longs-métrages de Jean-Daniel Pollet, Vincent Dieutre, Dominique Cabrera, Fleur Albert. Réalisateur, il a emprunté la voix de la fiction documentaire dès ses premiers films (Les Yeux brûlés, 1986, L'Impromptu de Jacques Copeau, 1993) avant de mettre en scène son personnage dans des «fantaisies documentaires » :J'ai quitté l'Aquitaine (2005), L'Emmuré de Paris (2018). Critique, Laurent Roth a fait partager sa passion pour le cinéma du réel par ses articles (Cahiers du Cinéma), ses chroniques (France Culture), ses livres (sur Abbas Kiarostami et Chris Marker), les festivals qu'il a dirigés (« Fictions du réel » à Marseille, «Cinécitoyen » à Paris).

\section{MICHÈLE VALENTIN}

Psychanalyste, spécialisée en psychopathologie et sémiologie, auteur de fables cliniques publiées dans la revue Enfance \& Psy chez Erès. Elle intervient dans le domaine de l'enfance et de la famille auprès des Juges des Enfants, dans le domaine de la précarité et du traumatisme avec différentes ONG, et en libéral dans le 12ème arrondissement, à Paris. 